\title{
Study protocol: a randomised controlled trial of a telephone delivered social wellbeing and engaged living (SWEL) psychological intervention for disengaged youth
}

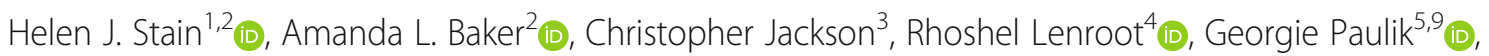
John Attia ${ }^{2,8}$ (D), Luke Wolfenden² (B), Stoyan R. Stoyanov ${ }^{6,7}$ (D), Holly Devir ${ }^{2}$ and Leanne Hides, ${ }^{6,7^{*}}$ (D)

\begin{abstract}
Background: Internationally, from 12.2-23.4\% of youth (aged 16-24 years) are not in employment, education or training (NEET). These disengaged youth are more likely to experience social exclusion, increased psychological distress and poor quality of life. Youth at risk of disengagement are less likely to access traditional support services, requiring development of innovative interventions.

Methods: The trial is a single blind, three arm, randomised controlled trial evaluating the effectiveness of a telephone delivered psychological intervention for disengaged youth (12-25 years). Participants will be randomised to receive either (i) SWEL, (ii) Befriending, or (iii) Single Session Psycho-Education. Therapy will be over an 8 week period with a minimum of four and maximum of eight sessions for the SWEL or Befriending conditions, or a single session for the Psycho-Education condition. Outcomes will be assessed at baseline and at 2, 8 and 14-month follow-up with the primary outcome being re-engagement in education, training or employment.

Discussion: This large, multi-site, randomised controlled trial will inform the delivery of services for young people at risk of disengaging from education or training. The provision of psychological therapy by telephone increases access by youth - especially those in rural and remote areas - both to the trial and the treatment, if adopted by services. The outcomes of this trial could have meaningful societal impact for a vulnerable population. It is expected that recruitment, intervention and retention will present challenges for the trial given the focus on disengaged youth.
\end{abstract}

Trial registration: ANZCTR, ACTRN12614001212640, Registered 18 Nov 2014. Retrospectively registered.

Ethics and dissemination: Ethics approval has been obtained from the participating institutions. Results of the trial will be submitted for publication in peer reviewed journals and findings presented at scientific conferences and to key service providers and policy makers.

Keywords: Social inclusion, NEET, Remote therapy, Youth services, CBT, DBT, Behavioural activation, Befriending, Motivational interviewing, Social wellbeing

\footnotetext{
* Correspondence: I.hides@uq.edu.au

${ }^{6}$ School of Psychology, University of Queensland, Brisbane, Australia

${ }^{7}$ School of Psychology, Queensland University of Technology, Brisbane,

Australia

Full list of author information is available at the end of the article
}

(c) The Author(s). 2019 Open Access This article is distributed under the terms of the Creative Commons Attribution 4.0 International License (http://creativecommons.org/licenses/by/4.0/), which permits unrestricted use, distribution, and reproduction in any medium, provided you give appropriate credit to the original author(s) and the source, provide a link to the Creative Commons license, and indicate if changes were made. The Creative Commons Public Domain Dedication waiver (http://creativecommons.org/publicdomain/zero/1.0/) applies to the data made available in this article, unless otherwise stated. 


\section{Background}

Young people with low educational attainment and/ or limited employment are more likely to experience social exclusion [1], increased psychological distress [2] and poor quality of life [3]. Currently, global youth unemployment reaches $13.1 \%$, three times that of adult rates [4] equating to nearly 75 million. Rates of youth (aged 16-24 years) not in employment, education or training (NEET) are reported at $23.4 \%$ in the European Union, $22.2 \%$ in the United Kingdom (UK), 15.5\% in United States of America, and $12.2 \%$ in Australia. Social exclusion, such as homelessness and unemployment, are associated with risk for mental health problems [2-5]. Australian Aboriginal youth continue to have poorer educational outcomes [6] and lower levels of social and emotional wellbeing compared to non-Aboriginal youth [7-9].

Cohort studies have shown that NEET youth are more likely to have mental health problems currently, or in childhood or adolescence, compared to non NEET youth [10], and that a history of mental health problems from childhood to young adulthood is associated with increased risk for NEET at age 19 [11]. The 2010 Australian National Strategy for Young Australians prioritises improving youth health and wellbeing by empowering young people in shaping their futures by securing education, increasing family support, encouraging community participation and providing early intervention [12]. Our clinical trial aims to improve young people's ability to make wise life choices such as participating in education.

The focus of the social well-being and engaged living intervention (SWEL) is on social engagement as a construct determined by social self-efficacy and interpersonal effectiveness. Social self-efficacy is the perceived ability of one's self to form and maintain social relationships [13], contributing to one's sense of agency or mastery [14]. According to Bandura [15], people with high self-efficacy are more likely to produce their own future rather than simply foretell it. This belief in the ability to alter one's own life circumstances is fundamental to our intervention. Results of a path analysis $(N=664$ adolescents) showed social self-efficacy beliefs influenced an adolescent's expectations for the future, sense of self, life satisfaction and positive emotions [13]. Self-efficacy beliefs have a direct positive impact on academic and social outcomes for adolescents through an enhanced sense of mastery [16] and have been associated with decreased likelihood of dropping out of school [17]. Interpersonal effectiveness can be measured by level and range of social activity, quality of relationships (independent of school or work engagement) and life satisfaction. SWEL has been specifically designed for this trial by integrating several evidence-based Cognitive Behavioral Therapy (CBT) and Dialectical Behaviour Therapy-derived therapies in order to target sense of self, interpersonal effectiveness and affect regulation. These abilities represent key developmental challenges for adolescents [16].

CBT has a strong evidence base across a range of ages and clinical disorders [18, 19]. Its delivery by internet, videoconference or telephone has the potential to increase access to psychological interventions, particularly for people with limited mobility (e.g. youth without their own transport) and rural residents. Trials of internet-delivered CBT have had mixed results. A review of online CBT interventions for anxiety or depression found that 23 out of 26 trials had clinically significant outcomes, with effect sizes of $0.42-0.65$ for depression and $0.29-1.74$ for anxiety [20]. A review of 20 clinical trials for anxiety and depression found that computerised CBT was as effective as therapist led CBT and more effective than treatment as usual (TAU) [21]. However another review of 12 randomised controlled trials (RCT) for anxiety and depression found large effect sizes for therapist supported online CBT compared to small effect sizes for online CBT alone [22]. Clinicians have reported the lack of therapist contact in internet delivered CBT for children and adolescents as concerning [23].

Telephone delivered CBT ensures therapist contact and flexibility with the intervention as the therapist can tailor the session according to the young person's presentation on the day. A meta-review of 13 remotely delivered RCTs for anxiety and depression included 10 trials of telephone-delivered psychotherapy and noted effect sizes consistent with published face to face trials [24]. A recent pilot study of telephone-delivered CBT for 10 adolescents with OCD found significant improvements in symptoms [25]. Feedback from the adolescents showed telephone CBT to be highly acceptable, convenient (less travel time), flexible (they could be away from home) and less stressful than attending a clinic [25]. Our research has shown similar results for psychological assessments conducted by videoconference compared to face to face [26].

\section{Objectives of the study}

This trial will determine the efficacy of a telephone delivered SWEL psychological intervention for improving the social engagement and emotional wellbeing of disengaged youth. It is hypothesised that participants receiving the SWEL intervention will achieve significantly higher levels of re-engagement in education, training or employment, than those receiving Befriending, or Single Session Psycho-Education. It is also hypothesised that mental wellbeing, self-esteem, social and occupational functioning, and affect regulation will be improved for 
participants receiving SWEL, while mental ill-health, alcohol and other drug use will be reduced.

\section{Methods Study design}

The trial is a single blind three arm randomised controlled trial evaluating the effectiveness of a telephone-delivered social well-being and engaged living (SWEL) intervention for improving the vocational, social and emotional functioning of disengaged youth. Participants will be randomised to receive either (i) SWEL, (ii) Befriending, or (iii) Single Session Psycho-Education. Befriending has been applied in a RCT of CBT for youth with a first episode of psychosis [27] and has been developed in earlier clinical trials to address the nonspecific elements of psychotherapy [28]. Outcomes will be assessed at baseline and at 2, 8 and 14-month follow-up, with assessments conducted over the phone and online. Figure 1 provides an overview of the trial participant flow. Design and implementation is being carried out with the ongoing direct input from our Aboriginal communities.

\section{Eligibility criteria}

Eligible participants will be aged $12-25$ years, and at risk of disengagement from education, training or employment, defined as non-attendance for at least 28 days in the past 3 months. Given our early intervention focus, young people will be excluded if they are chronically disengaged (> 6 months). This is consistent with the core or sustained (long term disengagement) subcategory for NEET young people in the UK [29]. Exclusions will include: severe intellectual disability and/or high dependence on medical care that may impede ability to re-engage with education, training or work; diagnosed psychotic disorder; hearing impairment; or insufficient English fluency to give informed consent. Those identified as being at high risk of suicide will be excluded until suicide risk is addressed. Thereafter participants will be invited to be rescreened for eligibility.

\section{Recruitment and follow up procedures}

Participants will be recruited through youth employment, educational and vocational training services in two Australian States: Queensland and New South Wales. These include three YMCA Vocational Schools in Brisbane and Ipswich, Yourtown (formerly Boystown), several secondary schools in Newcastle and Toowoomba Youth Service. Research team members will regularly visit each site to explain the study to staff and young people, attend the first week of vocational group programs and parent/teacher interviews, and regularly phone/email the sites to check for referrals. Parents/ guardians of participants aged under 18 years will be provided a copy of the parent/guardian information statement and will be asked to confirm that the young person is capable of understanding the study and of consenting to take part. Thereafter, parents/ guardians will be able to return the signed consent form, or to verbally consent over the phone to the recruitment team. The requirement for parent/guardian consent will not be enforced if the young person is considered to be a 'mature minor' that lives/functions independently of their parent/guardian or does not wish their parent/guardian to be involved as this may cause psychological distress or psychosocial harm. The research psychologist will determine if the young person is of sufficient age and maturity to provide informed consent based on their ability to understand and recall the aims, potential risks and benefits of the research. An Aboriginal Youth Officer will facilitate the referral of Aboriginal youth to the project. Sites will complete a referral form or provide contact details. Self-referrals will also be accepted. Recruitment will also be conducted via Facebook and an age-targeted advertisement on the university's online research page. By clicking on the advertisement, young people will be taken to an online participant information sheet and self-referral form, submittable to the research team.

SWEL research clinical psychologists will contact new participants within $24 \mathrm{~h}$ of receiving referrals. They will provide detailed information about the trial, obtain consent verbal and confirm eligibility. A 20-30 min baseline assessment will be conducted by phone immediately after screening. During this assessment participant age, gender, ethnicity and location will be recorded. Thereafter participants will complete online versions of self-report measures. Reporting will follow the CONSORT guidelines (Fig. 1) [30]. Follow-up assessments consisting of an online survey and telephone interview will be conducted at 2,8 , and 14 months post-baseline by research officers blinded to treatment allocation. To enhance retention, participants will be emailed a link to the online survey 1 day before, then one and 3 days after the follow-up is due. In order to ensure that participants have received the survey link, it will also be sent via a SMS 2 days after the online survey is due. Participants will receive \$AUD40 cash for each assessment time point (total \$AUD160) to encourage engagement and follow-up completion. Those who have not responded to the initial calls will be contacted on a weekly basis by phone and SMS for up to 4 weeks after follow-up is due.

\section{Randomisation}

Randomisation will be performed after the baseline survey by an automated web-based research trials management system. Participant allocation will be concealed in a secure database within the program, 
accessible only by the website manager. It will be generated with permuted blocks of varying sizes and stratified by age (12-18 or $19-25$ years old), gender, zone (rural or urban) and indigenous status (yes or no), to allocate participants to one of the three treatment groups.

\section{Measures}

Outcome measures are limited to $30 \mathrm{~min}$ for completion to minimise assessment fatigue and are completed online or by telephone. The assessment schedule is provided in Table 1.

\section{Screening measures}

Engagement screener The 2-item Brief Time Use Questionnaire was developed for this study to measure level of engagement in education, training, employment and job seeking. Participants report the date they last attended school, work or training, and the number of attendance days over the past 6 months to assess inclusion criteria for recent disengagement. They report the

\section{Enrolment}

Allocation

Received referral information $(\mathrm{N}=)$

Eligible/Potentially eligible $(\mathrm{n}=)$

Telephone screen $(n=; 93 \%$ of those eligible)

Excluded $(\mathrm{n}=)$

Not meeting inclusion criteria Refused to participant Other reasons ( $n=; 93 \%$ of those eligible)

Intervention

PSYCHOEDUCATION

$(n=93)$

1 session completed within 9 weeks

Follow-up

Follow-up

Post-treatment

6 month

12 month

Analysis

Analysed $(\mathrm{N}=)$

Excluded $(\mathrm{N}=)$

Fig. 1 Consort diagram

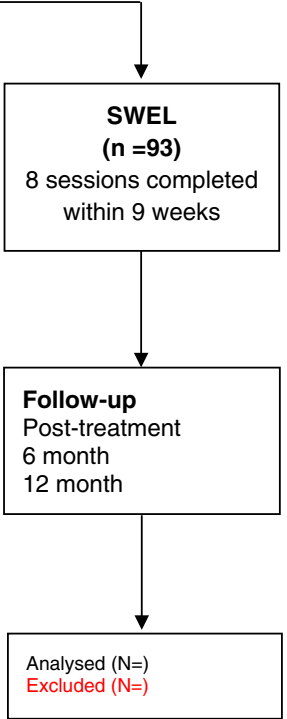

Withdrawn after Screen (before randomisation) $(\mathrm{n}=)$

BEFRIENDING

$$
\text { ( } n=93 \text { ) }
$$

8 sessions completed within 9 weeks

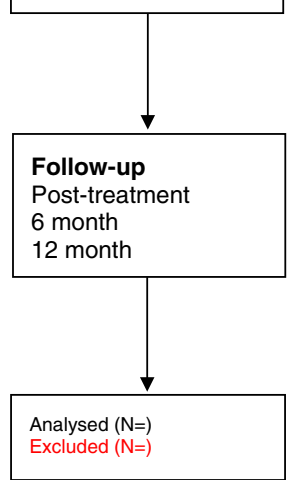

Excluded ( $\mathrm{N}=$ 
Table 1 Assessment Schedule

\begin{tabular}{|c|c|c|c|c|c|}
\hline Instrument & Domain & $\mathrm{BL}$ & $2 \mathrm{~m}$ & $8 \mathrm{~m}$ & $14 \mathrm{~m}$ \\
\hline Engagement: a Brief Time Use Questionnaire & Vocational engagement & $\checkmark$ & & & \\
\hline Psychosis Screener & Psychosis & $\checkmark$ & & & \\
\hline MINI Suicidality Scale & Suicidality & $\checkmark$ & & & \\
\hline Demographics Accommodation, Financial support & Demographics & $\checkmark$ & $\checkmark$ & $\checkmark$ & $\checkmark$ \\
\hline Engagement: a Comprehensive Time-Use Questionnaire & Vocational engagement & $\checkmark$ & $\checkmark$ & $\checkmark$ & $\checkmark$ \\
\hline Multidimensional Adolescent Functioning Scale (MAFS) & Functioning & $\checkmark$ & $\checkmark$ & $\checkmark$ & $\checkmark$ \\
\hline Social \& Occupational Functioning Assessment Scale (SOFAS) & Social and Occupational Functioning & $\checkmark$ & $\checkmark$ & $\checkmark$ & $\checkmark$ \\
\hline Global Functioning Social \& Global Functioning Role Scales & Social and Role Functioning & $\checkmark$ & $\checkmark$ & $\checkmark$ & $\checkmark$ \\
\hline Social Functioning Scale of the Lehman Quality of Life Scale & Social activity, quality of life, relationship quality & $\checkmark$ & $\checkmark$ & $\checkmark$ & $\checkmark$ \\
\hline Mental Health Continuum Short Form & Mental wellbeing & $\checkmark$ & $\checkmark$ & $\checkmark$ & $\checkmark$ \\
\hline Kessler 10 (K10) & Mental ill-health & $\checkmark$ & $\checkmark$ & $\checkmark$ & $\checkmark$ \\
\hline Brief Resilience Scale & Resilience & $\checkmark$ & $\checkmark$ & $\checkmark$ & $\checkmark$ \\
\hline Regulation of Emotions Questionnaire & Emotional wellbeing & $\checkmark$ & $\checkmark$ & $\checkmark$ & $\checkmark$ \\
\hline WHO-ASSIST & Substance use & $\checkmark$ & $\checkmark$ & $\checkmark$ & $\checkmark$ \\
\hline Perceived Empathic and Social Efficacy Scale & Social engagement & $\checkmark$ & $\checkmark$ & $\checkmark$ & $\checkmark$ \\
\hline Rosenberg Self Esteem Scale & Self esteem & $\checkmark$ & $\checkmark$ & $\checkmark$ & $\checkmark$ \\
\hline Treatment Recording Form & Other treatment received & & $\checkmark$ & $\checkmark$ & $\checkmark$ \\
\hline Youth Therapy Satisfaction Questionnaire & Treatment satisfaction & & $\checkmark$ & & \\
\hline
\end{tabular}

number of attendance days for each month, going backwards from the day of the survey (excluding school holidays). A ratio of engagement is calculated as a percentage based on actual days attended/days required to attend. This allows for a correct calculation of engagement, accounting for different study or work schedule requirements. If a participant's ratio of attendance is less than two thirds in the last 3 months (i.e. they missed at least one third of the days of school/work/training in the last 3 months) then they are considered 'disengaged' and eligible for the program. If a total of zero days of attendance are reported over the past 6 months, participants are considered 'too disengaged' and excluded from the study. If more than two thirds of the days of attendance is reported over the past 3 months then participants are also excluded as they are considered 'too engaged'.

Active psychosis The 7-item Psychosis Screener from the Composite International Diagnostic Interview (CIDI) will be used to assess for the presence of characteristic psychotic symptoms [31].

Suicidal ideation The 6-item Suicidality Scale of the Mini International Neuropsychiatric Interview (MINI) will be used to assess suicide risk [32].

Based on eligibility, a skip-logic implemented at the end of the screening survey leads directly into the Baseline survey, or to the end of the survey.

\section{Baseline and follow-up measures}

Demographics Age, gender, ethnicity, living arrangements, financial support, years of education and relationship status.

Engagement A Comprehensive Time-Use Questionnaire of 28 items with skip logic measures the level of engagement in education, training, employment and seeking work since the last follow-up. Items include type of education attended (e.g. school, certificate, etc.), number of hours attended and length of enrolment in the program. The items for training, employment, job searching and volunteer work follow the same pattern.

Functioning The Multidimensional Adolescent Functioning Scale (MAFS) is a 23-item self-report measure of general, family and peer functioning [33]. Social and occupational functioning is measured using the Social and Occupational Functioning Assessment Scale (SOFAS) [34] and the Global Functioning: Social (GF-Social) and Global Functioning: Role (GF-Role) scales [35, 36]. Social activity, quality of life and relationship quality are measured by the Social Functioning Scale (SFS) of the Lehman Quality of Life Scale [37].

Mental ill-health and wellbeing Self-report information on the young person's mental health history is collected including current mental health treatment, and family history of mental health problems. The Mental 
Health Continuum Short Form is a 14-item positive mental health scale measuring emotional, psychological and social wellbeing [38]. The Kessler 10 (K10) [39] is a 10-item self-report questionnaire measuring psychological distress in the past month. Normative data indicates a cut-off of $\geq 17$ is at the 75th percentile among Australian youth [40]. The young person's ability to bounce back or recover from stress is measured by the 6-item Brief Resilience Scale [41].

Affect regulation The Regulation of Emotions Questionnaire [42] (21 items) was developed and tested in a sample of adolescents measuring adaptive and maladaptive strategies for processing emotions.

Alcohol and other drug use The 8-item Alcohol, Smoking and Substance Involvement Screening Test Version 3.0 (ASSIST) [43] measures lifetime and recent (past 3 months) use of 10 substances, as well as abuse and dependence symptoms. It was developed by the World Health Organisation (WHO) as a screening instrument for all psychoactive substances, with high levels of internal consistency, construct, concurrent and discriminant validity [44].

Self-efficacy The Perceived Empathic and Social Efficacy Scale is an 11-item measure that assesses self-efficacy beliefs regarding both empathic responding to others' needs or feelings, and managing interpersonal relationships [45]. The Rosenberg Self-Esteem Scale is a 10-item measure of global self-esteem [46].

\section{Psychological interventions}

Research therapists will complete a one-day training workshop [led by HS and/or LH] for the SWEL, Befriending and Single Session Psycho-Education conditions. An Aboriginal Youth Worker will be available to support the research therapists and the Aboriginal participants with treatment engagement, attendance and delivery. All interventions will be delivered by telephone and participants will be reimbursed \$AUD10 cash per session to maximise retention and treatment completion. Participants in the SWEL and Befriending conditions will initially be encouraged to participate in at least four sessions. Once engaged they will be offered four additional sessions, receiving a maximum of eight (one per week). Participants randomised to Psycho-Education will receive one session over the eight-week period. Sessions are 30-60 min duration and will be audio-recorded. Therapists will ensure participants are in an appropriate setting during therapy calls to maintain participant privacy and confidentiality.

\section{SWEL intervention}

The SWEL manualised intervention is designed to enhance young people's vocational engagement by targeting their emotional and social self-efficacy. It has a CBT framework and is divided into two sets of modules. The core modules are delivered to all participants in four sessions. Thereafter, participants are offered four further sessions on a needs basis, to revisit core modules and/or add optional modules. The four core modules are assessment, formulation/goal setting, emotion regulation/ interpersonal effectiveness, and behavioural activation/ engaged living. The three optional modules are self-esteem, problem solving, and drug and alcohol use using motivational interviewing. The final session serves for review and consolidation of skills.

\section{Befriending intervention}

Befriending is a manualised intervention developed in earlier clinical trials to address the non-specific elements of psychotherapy [47]. It controls for therapist contact time, client expectancies, therapeutic alliance and therapist factors (e.g., warmth and understanding) and has been used in a randomised controlled trial of CBT for youth with a first episode of psychosis [28]. It does not apply therapeutic techniques specific to major models of psychotherapy and focuses on everyday events and topics using a conversational, friendly approach, without problem solving or examination of emotions. Befriending will be delivered for up to eight weekly sessions to allow for the same level of contact as the SWEL condition.

\section{Single session psycho-education plus TAU intervention}

This single-session intervention was specifically developed for this study to deliver feedback from the baseline assessment and information on relevant topics for disengaged youth including wellbeing, depression, anxiety, anger and substance use. The session will be delivered using a conversational approach.

\section{Treatment satisfaction and fidelity}

Treatment satisfaction will be assessed at 2-month follow up using the 7-item Youth Therapy Satisfaction Questionnaire. This adaptation of the Youth Satisfaction Questionnaire [48] includes two additional questions asking if the participant would recommend telephone counselling to a friend and if they prefer telephone to face to face counselling. All treatment sessions will be audio-recorded and session component checklists will be completed following each session for controlling the content and dose of therapy each participant receives. A random sample (20\% of participants; evenly spread across the three treatment groups) of session recordings 
will be independently rated for treatment fidelity using an adapted version of the ACE Treatment Integrity Measure (ATIM) [49]. Every treatment session delivered to the $20 \%$ of participants selected will be rated. The session checklists will also be completed to confirm the content and dose of treatment delivered.

\section{Adaptation of the ATIM [49]}

The ATIM was chosen to measure treatment integrity as it was designed to detect adherence and differentiation between CBT and Befriending. Since CBT treatments differ in their emphasis on specific CBT techniques, it is important that treatment fidelity measures be tailored to the treatment manual $[50,51]$. Consequently, the ATIM was adapted to the specific treatment manuals used in the present study. This resulted in the addition of five SWEL strategy items (Therapist provides assessment feedback; Therapist works with client to identify client's strengths/positive qualities; Therapist reviews and summarises session or recaps on previous session; Therapist guides client in Behavioural Activation; Therapist introduces session/sets agenda), one befriending strategy item (Client and therapist engage in neutral conversations about day to day topics), one general therapy technique item (Therapist does suicide risk assessment) and one item to assess differentiation between three interventions rather than two (i.e., CBT, Befriending and Psycho-Education). Three items were also removed because they involved strategies not used in the present study (Therapist and client work on thought records; Therapist and client engage in role reversal exercises; Therapist engages client in exposure training).

A competence measure was also added to the ATIM as this is a key component of treatment integrity [5052]. For each strategy endorsed as present in each session, the following therapist competence item was added, "How competent was the therapist at delivering this component" rated on a 7-point Likert scale (Very Poor to Excellent). The item was based on competence items used in the established Yale Adherence and Competence Scale [53]. The final 69-item measure consists of 34 items for assessment of adherence, 34 for competence, and one item asking the rater to guess which therapy was delivered. Six of the adherence items target Befriending, 23 target SWEL and five target general therapy techniques (e.g., collaboration, empathy, professionalism).

\section{Assessment integrity}

Baseline and follow up assessments will be audio recorded. Reliability and adherence to protocol will be assessed by an independent researcher on a random sample of $20 \%$ of participants. Research officers will receive fortnightly supervision to monitor assessment, retention and blinding. The chief investigators, follow-up assessor and trial statistician will be blind to treatment group allocation.

\section{Sample size calculation}

A total of 279 youth (93 individuals per group) will be recruited. Allowing for a 30\% loss to follow-up, the trial will have $80 \%$ power and a $5 \%$ type I error to find a Cohen's $d$ of 0.5 , i.e. moderate effect size. The significance level is adjusted by a factor of 2 to control the overall type I error rate at $5 \%$ for the 2 pair-wise comparisons required to test the primary hypotheses. This estimate is conservative and does not take into account the added power from adjusting for an individual's measure at baseline or the repeated measures of the outcomes.

\section{Statistical analysis}

The primary outcome of this study will be engagement in education, training or employment (Time-Use Questionnaire) at the 2 month time point. Secondary outcomes include the Time-Use Questionnaire at 8 and 14 months, as well as social activity, quality of life and relationship quality (SFS), perceived social self-efficacy (Perceived Empathic and Social Efficacy Scale), sense of self (Rosenberg Self-Esteem Scale), and functioning (SOFAS, GF: Social, GF- Role, SFS, MAFS), measured at baseline, end of treatment ( 2 months) and at 6 and 12 months post treatment.

The primary hypothesis is that the overall engagement score (calculated as actual/expected engagement in school/ work/training) of youth who are randomised to active treatment (SWEL) will be higher than those randomised to either Befriending or Psycho-Education at the 2 month time point. Differences between treatment groups will be tested within a Linear Mixed Model (LMM). The outcome in the model will be individual total engagement scores at each post treatment time point and the predictor variables in the model will include treatment group, time, the interaction between treatment group and time, baseline engagement score and the stratifying variables. The model will include a random intercept term to control for the repeated measurements on individuals. All analyses will be based on the intention to treat principle. Differences between groups in all secondary outcomes will be tested using the same approach as outlined for the primary outcome variable. Additional LMM will be used to test for a difference between treatment groups in the trend of each of these outcomes from baseline to 12 months post-treatment using data from all time points. Subgroup analyses will be conducted to examine the similarity of the treatment effect across age groups.

\section{Trial duration}

July 2014 - June 2018. 


\section{Ethics and dissemination}

Safety and risk management protocols were devised to manage safety or urgent treatment issues. Young people recruited to this study and subsequently identified as needing more intensive treatment will be appropriately referred. A clinical committee comprised of $\mathrm{LH}, \mathrm{AB}$, the project manager, research clinicians and follow-up researchers will meet fortnightly to resolve treatment difficulties and discuss any departures from the protocol. The authors will meet quarterly to monitor the study's implementation, clinical and research integrity. The informed consent of young people will be obtained by recruitment staff and parental or guardian consent for people aged $12-15$ years will be sought.

\section{Discussion}

This study protocol describes a large, multi-site, randomised controlled trial of a telephone-delivered psychological intervention for improving the social engagement and emotional wellbeing of young people who are disengaging from education, employment or training. The engagement of young people in completion of education or training is a major economic and societal concern in many countries. Internationally, many governments have distributed short term funding to non-government organisations in an effort to address this issue. However, there is a lack of an evidence base to guide interventions for disengaged youth and the intermittent nature of funding for non-government organisations challenges the sustainability of services.

The SWEL study aims to determine the effectiveness of a telephone delivered psychological intervention in improving the engagement of young people in education, employment or training. The provision of psychological therapy by telephone increases access by youth - especially those in rural and remote areas - both to the trial and the treatment, if adopted by services. It is hypothesised that young people receiving the SWEL intervention will achieve significantly higher levels of reengagement in education, training or employment, than those receiving Befriending, or Single Session Psycho-Education. It is also hypothesised that mental wellbeing, self-esteem, social and occupational functioning, and affect regulation will be improved for participants receiving SWEL, while mental ill-health, alcohol and other drug use will be reduced. The outcomes of this trial could have meaningful societal implications for a vulnerable population. Recruitment to the trial was delayed following the loss of national funding to the referral services but commenced in July 2014 and will end in June 2018.

\section{Acknowledgements}

The authors would like to acknowledge the contribution of Dr. Patrick McElduff and Dr. Jennifer Connoly made to this research project.

\section{Funding}

This study was funded by an Australian National Health and Medical Research Council Targeted Call for Mental Health Research Project Grant (APP1044507)

Availability of data and materials

Not applicable.

\section{Authors' contributions}

$\mathrm{HS}$ prepared the body of the protocol. $\mathrm{AB}$ contributed to the preparation of the entire body of the protocol. CJ contributed to the preparation of the entire body of the protocol. RL contributed to the preparation of the entire body of the protocol. GP contributed to the preparation of the entire body of the protocol. JA prepared all information regarding sample size and statistical analysis. LW contributed to the preparation of the entire body of the protocol. SS prepared study flow description, assessment description, table, diagram, references and formatted the manuscript. HD contributed to the preparation of the entire body of the protocol. LH contributed to the preparation of the entire body of the protocol. All authors read and approved the final manuscript.

Ethics approval and consent to participate

Reviewed by Queensland University of Technology, Ethics approval number: 1300000403. Participants and/or parents/guardians will consent by signing the consent form, or by verbally consenting over the telephone.

Consent for publication

Not applicable.

\section{Competing interests}

The authors declare that they have no competing interests.

\section{Publisher's Note}

Springer Nature remains neutral with regard to jurisdictional claims in published maps and institutional affiliations.

\section{Author details}

${ }^{1}$ School of Social and Health Sciences, Leeds Trinity University, Leeds, UK. ${ }^{2}$ School of Medicine and Public Health, University of Newcastle, Newcastle, Australia. ${ }^{3}$ Early Intervention Service, Birmingham and Solihull NHS Foundation Trust, Birmingham, UK. ${ }^{4}$ School of Psychiatry, University of New South Wales, Sydney, Australia. ${ }^{5}$ School of Psychology and Exercise Science, Murdoch University, Perth, Australia. ${ }^{6}$ School of Psychology, University of Queensland, Brisbane, Australia. ${ }^{7}$ School of Psychology, Queensland University of Technology, Brisbane, Australia. ${ }^{8}$ Hunter Medical Research Institute, Newcastle, Australia. ${ }^{9}$ Perth Voices Clinic, Perth, Australia.

Received: 24 September 2018 Accepted: 12 April 2019

Published online: 06 May 2019

\section{References}

1. Wanberg CR. The individual experience of unemployment. Annu Rev Psychol. 2012;63(1):369-96.

2. Bynner J, Parsons S. Social exclusion and the transition from school to work: the case of young people not in education, employment, or training (NEET). J Vocat Behav. 2002;60(2):289-309.

3. Mustard CA, Bielecky A, Etches J, Wilkins R, Tjepkema M, Amick BC, et al Mortality following unemployment in Canada, 1991-2001. BMC Public Health. 2013:13(1):441.

4. International Labour Office. Global employment trends 2014: risk of a jobless recovery? Geneva: ILO; 2014

5. Baggio S, Iglesias K, Deline S, Studer J, Henchoz Y, Mohler-Kuo M, Gmel G. Not in education, employment, or training status among young Swiss men. Longitudinal associations with mental health and substance use. J Adolesc Health. 2015;56(2):238-43

6. Silburn SR, Blair E, Griffin JA, Zubrick SR, Lawrence DM, Mitrou FG, et al. Developmental and environmental factors supporting the health and wellbeing of aboriginal adolescents. Int J Adolesc Med Health. 2007:19(3):345-54

7. Sawyer MG, Guidolin M, Schulz KL, McGinnes B, Zubrick SR, Baghurst PA. The mental health and wellbeing of adolescents on remand in Australia. Aust NZJ Psychiatry. 2010;44(6):551-9. 
8. Dingwall KM, Cairney S. Psychological and cognitive assessment of indigenous Australians. Aust NZJ Psychiatry. 2010;44(1):20-30.

9. Thomas A, Cairney S, Gunthorpe W, Paradies Y, Sayers S. Strong souls: development and validation of a culturally appropriate tool for assessment of social and emotional well-being in indigenous youth. Aust NZJ Psychiatry. 2010:44(1):40-8.

10. Goldman-Mellor S, Caspi A, Arseneault L, Ajala N, Ambler A, Danese A, et al. Committed to work but vulnerable: self-perceptions and mental health in NEET 18-year olds from a contemporary British cohort. J Child Psychol Psychiatry. 2016;57(2):196-203.

11. Veldman K, Reijneveld SA, Ortiz JA, Verhulst FC, Bültmann U. Mental health trajectories from childhood to young adulthood affect the educational and employment status of young adults: results from the TRAILS study. Epidemiol Community Health. 2015;69(6):588-93.

12. Australian Government. National strategy for young Australians. Canberra: Australian Government; 2010.

13. Caprara GV, Steca P, Gerbino M, Paciello M, Vecchio GM. Looking for adolescents' well-being: self-efficacy beliefs as determinants of positive thinking and happiness. Epidemiol Psychiatr Sci. 2006;15(1):30-43.

14. Bandura A. Social cognitive theory: an agentic perspective. Annu Rev Psychol. 2001:52(1):1-26.

15. Bandura A. Self-efficacy mechanism in human agency. Am Psychol. 1982; 37(2):122-47.

16. Bandura A, Caprara GV, Barbaranelli C, Gerbino M, Pastorelli C. Role of affective self-regulatory efficacy in diverse spheres of psychosocial functioning. Child Dev. 2003;74(3):769-82.

17. Theunissen $\mathrm{M}-\mathrm{J}$, Bosma H, Verdonk P, Feron F. Why wait? Early determinants of school dropout in preventive pediatric primary care. PLoS One. 2015; 10(11):e0142315.

18. Hides L, Samet S, Lubman DI. Cognitive behaviour therapy (CBT) for the treatment of co-occurring depression and substance use: current evidence and directions for future research. Drug Alcohol Rev. 2010;29(5):508-17.

19. Rathod S, Phiri P, Kingdon D. Cognitive behavioral therapy for schizophrenia. Psychiatr Clin North Am. 2010;33(3):527-36.

20. Griffiths KM, Farrer L, Christensen $H$. The efficacy of internet interventions for depression and anxiety disorders: a review of randomised controlled trials. Med J Aust. 2010;192(11):S4.

21. Kaltenthaler E, Brazier J, De Nigris E, Tumur I, Ferriter M, Beverley C, et al. Computerised cognitive behaviour therapy for depression and anxiety update: a systematic review and economic evaluation. Health Technol Assess. 2006;10(33):1-168.

22. Spek V, Cuijpers P, Nyklíček I, Riper H, Keyzer J, Pop V. Internet-based cognitive behaviour therapy for symptoms of depression and anxiety: a meta-analysis. Psychol Med. 2007;37(3):319-28.

23. Stallard P, Richardson T, Velleman S. Clinicians' attitudes towards the use of computerized cognitive behaviour therapy (CCBT) with children and adolescents. J Behav Cogn Psychother. 2010;38(5):545-60.

24. Bee PE, Bower P, Lovell K, Gilbody S, Richards D, Gask L, et al. Psychotherapy mediated by remote communication technologies: a meta-analytic review. BMC Psychiatry. 2008;8(1):60

25. Turner C, Heyman I, Futh A, Lovell K. A pilot study of telephone cognitivebehavioural therapy for obsessive-compulsive disorder in young people. J Behav Cogn Psychother. 2009;37(4):469-74.

26. Stain HJ, Payne K, Thienel R, Michie P, Carr V, Kelly B. The feasibility of videoconferencing for neuropsychological assessments of rural youth experiencing early psychosis. J Telemed Telecare. 2011;17(6):328-31.

27. Jackson H, McGorry P, Killackey E, Bendall S, Allott K, Dudgeon P, et al. Acute-phase and 1-year follow-up results of a randomized controlled trial of CBT versus befriending for first-episode psychosis: the ACE project. Psychol Med. 2008;38(5):725-35.

28. Sensky T, Turkington D, Kingdon D, Scott JL, Scott J, Siddle R, et al. A randomized controlled trial of cognitive-behavioral therapy for persistent symptoms in schizophrenia resistant to medication. Arch Gen Psychiatry. 2000:57(2):165-72

29. Allen T, Mehta P, Rutt S. Hidden talents: a statistical overview of the participation patterns of young people aged 16-24. Slough: National Foundation for Educational Research (NFER); 2012. p. 16-24.

30. Boutron I, Moher D, Altman DG, Schulz KF, Ravaud P. Methods and processes of the CONSORT group: example of an extension for trials assessing nonpharmacologic treatments. Ann Intern Med. 2008. 148(4):W60-6.
31. Degenhardt L, Hall W, Korten A, Jablensky A. Use of a brief screening instrument for psychosis: results of an ROC analysis. New South Wales: National Drug and Alcohol Research Centre (NDARC); 2005. p. 1-18.

32. Sheehan DV, Lecrubier $Y$, Sheehan $\mathrm{KH}$, Amorim $\mathrm{P}$, Janavs J, Weiller $\mathrm{E}$, et al. The mini-international neuropsychiatric interview (M.I.N.I.): the development and validation of a structured diagnostic psychiatric interview for DSM-IV and ICD-10. J Clin Psychiatry. 1998;59(Suppl 20):22-33.

33. Wardenaar K, Wigman J, Lin A, Killackey E, Collip D, Wood S, et al. Development and validation of a new measure of everyday adolescent functioning: the multidimensional adolescent functioning scale. J Adolesc Health. 2013;52(2):195-200.

34. American Psychiatric Association. Task Force on D-I. Diagnostic and statistical manual of mental disorders IV text revision (DSM-IV-TR). Washington: American Psychiatric Association; 2000.

35. Auther A, Smith C, Cornblatt B. Global functioning: social scale (GF: Social). Glen Oaks: Zucker-Hillside Hospital; 2006.

36. Niendam T, Bearden C, Johnson J, Cannon T. Global functioning: role scale (GF: role). Los Angeles: University of California; 2006.

37. Lehman AF, Ward NC, Linn LS. Chronic mental patients: the quality of life issue. Am J Psychiatry. 1982;139(10):1271-6.

38. Lamers S, Westerhof GJ, Bohlmeijer ET, Ten Klooster PM, Keyes CL. Evaluating the psychometric properties of the mental health continuumshort form (MHC-SF). J Clin Psychol. 2011;67(1):99-110.

39. Kessler RC, Barker PR, Colpe L, Epstein JF, Gfroeter JC, Hiripi E, et al. Screening for serious mental illness in the general population. Arch Gen Psychiatry. 2003;60(2):184-9.

40. Slade T, Grove R, Burgess P. Kessler psychological distress scale: normative data from the 2007 Australian National Survey of mental health and wellbeing. Aust NZJ Psychiatry. 2011:45(4):308-16.

41. Smith BW, Dalen J, Wiggins K, Tooley E, Christopher P, Bernard J. The brief resilience scale: assessing the ability to bounce back. Int J Behav Med. 2008; 15(3):194-200.

42. Phillips K, Power M. A new self-report measure of emotion regulation in adolescents: the regulation of emotions questionnaire. Clin Psychol Psychother. 2007;14(2):145-56.

43. WHO ASSIST Working Group. The alcohol, smoking and substance involvement screening test (ASSIST): development, reliability and feasibility. Addiction. 2002;97(9):1183-94.

44. Humeniuk R, Ali R, Babor TF, Farrell M, Formigoni ML, Jittiwutikarn J, et al. Validation of the alcohol, smoking and substance involvement screening test (ASSIST). Addiction. 2008;103(6):1039-47.

45. Di Giunta L, Eisenberg N, Kupfer A, Steca P, Tramontano C, Caprara GV. Assessing perceived empathic and social self-efficacy across countries. Eur J Psychol Assess. 2010;26(2):77-86.

46. Tomas JM, Oliver A. Rosenberg's self-esteem scale: two factors or method effects. Struct Equ Modeling. 1999;6(1):84-98.

47. Bendall S, Killackey E, Jackson H, Gleeson J. Befriending manual. Melbourne: ORYGEN Research Centre, University of Melbourne; 2003.

48. Stuntzner-Gibson D, Koren PE, DeChillo N. The youth satisfaction questionnaire: what kids think of services. Fam Soc. 1995;76(10):616.

49. Bendall S, Allott K, Jovev M, Marois M-J, Killackey EJ, Gleeson JF, et al. Therapy contamination as a measure of therapist treatment adherence in a trial of cognitive behaviour therapy versus befriending for psychosis. J Behav Cogn Psychother. 2015;43(3):314-27.

50. Rollinson R, Smith B, Steel C, Jolley S, Onwumere J, Garety PA, et al. Measuring adherence in CBT for psychosis: a psychometric analysis of an adherence scale. J Behav Cogn Psychother. 2008;36(2):163-78.

51. Perepletchikova F, Kazdin AE. Treatment integrity and therapeutic change: issues and research recommendations. Clin Psychol. 2005;12(4):365-83.

52. Waltz J, Addis ME, Koerner K, Jacobson NS. Testing the integrity of a psychotherapy protocol: assessment of adherence and competence. J Consult Clin Psychol. 1993;61(4):620-30.

53. Nuro KF, Maccarelli L, Baker SM, Martino S, Rounsaville B, Carroll K. Yale adherence and competence scale (YACSII) guidelines. West Haven: Yale University Psychotherapy Development Centre; 2005. p. 161. 